\title{
STAINING OF THE INCLUSION BODIES OF TRACHOMA AND INCLUSION CONJUNCTIVITIS*
}

\author{
BY
}

\author{
M. J. GILKES, C. H. SMITH, AND J. SOWA \\ Trachoma Research Group, Medical Research Council
}

THE first description of the inclusion bodies of trachoma was made by Halberstaedter and von Prowazek following the examination of Giemsa-stained scrapings from the conjunctivae of baboons which had been inoculated with secretions from trachomatous patients ( $c f$. Julianelle, 1938). Since then Giemsa and its modifications have held prime place as a means of demonstrating these structures and this position has been upheld by the recommendation of its use in the Second Report of the WHO Expert Committee on Trachoma (1955): "for trachoma studies-for the examination of smears and scrapings from the conjunctiva".

Although Giemsa may well constitute a standard reference of staining, certain problems arise from its use: first, the prolonged staining time and the need for solutions of controlled $\mathrm{pH}$; secondly, the superficial similarity between the Giemsa-stained HP inclusion and a number of other intracellular appearances (e.g. granular cytoplasm, nuclear prolapse), which, even in experienced hands, militates against the speedy examination of smears; thirdly, the difficulty of detecting HP inclusions in thick clumps of tissue; finally, the difficulty in distinguishing the full details of inclusion morphology when this overlies a deeply-stained cell nucleus.

These factors have led to a search for suitable alternative stains. Among these are Wright's modification of Giemsa, Castaneda's stain, Lindner's contrast stain, Macchiavello's stain, and Poleff's stain. Of these most have proved of no greater value than Giemsa, while others, such as Poleff's methylene blue, have been found unreliable (Thygeson, 1955), owing to the ease with which various intracytoplasmic structures may simulate HP inclusions.

Despite the recommendation in the First Report of the WHO Expert Committee on Trachoma (1952) "... the development of rapid methods for the detention of inclusion bodies: Thick scrapings. Iodine stain ...", little further work appears to have been done, except that of Dark (1955), confirming the earlier studies of Rice (1936) and Thygeson (1938), on the staining by iodine of the glycogen-containing matrix of the HP inclusion.

During the course of a recent project on the aetiology of trachoma this problem of speedy and accurate detection assumed prominence as it was

* Received for publication February 28, 1958. 
necessary to demonstrate the presence of HP inclusions in conjunctival scrapings, before their inoculation into tissue culture. In the case of negative material, full microscopy of Giemsa-stained smears from both eyes was found to require up to one hour per slide. The possibility of inclusionpositive cases being discarded owing to inclusions being missed in thick clumps of tissue was also present. Following unsatisfactory trials of the Castaneda and Macchiavello stains, it was decided to experiment with the iodine-staining method of Rice (1936).

\section{Methods}

In our hands the technique reported by Rice (1936) and Thygeson (1938) proved unsatisfactory. The staining was faint and liable to fade. Acidification of the iodine solution, advocated by Rice, gave no improvement.

Some of the difficulty lay in the strength of the Lugol's solution-in the earlier reports the strength is not stated. Gatenby (1956) has enumerated the various strengths of solution which have been described under this title, ranging from 0.33 to 5 per cent. iodine in aqueous, or even alcoholic, solutions of potassium iodide from 2 to 10 per cent. It would seem probable that this confusion has resulted in other workers using insufficient concentrations with correspondingly disppointing differentiation of HP inclusions. Initially we had been using bacteriological Lugol ( 0.33 per cent. $\mathrm{I}_{2}$ in 2 per cent. $\mathrm{KI}$ aqueous solution) but transfer to clinical Lugol ( 5 per cent. $\mathrm{I}_{2}$ in 10 per cent. KI aq.) yielded much more favourable results.

The use of unfixed material was found to give greatly improved results in terms of definition, density and duration of staining with no tendency for the scraping to become detached from the slide, presumably owing to the fixative action of the iodine. Alcohol fixation was found to have a particularly deleterious effect and the presence of small amounts, even as vapour, after staining, caused rapid decolourization.

After further investigation a final technique was evolved, and this has been used on several hundreds of slides of scrapings from trachomatous patients in Jordan and Gambia. The appearances reported have also been found to be valid in three cases of inclusion conjunctivitis seen in the U.K.

First, scrapings are smeared on clean dry slides and allowed to dry in the air for at least 5 minutes; they are covered with 5 per cent. iodine in 10 per cent. KI aqueous Lugol's solution which is left in situ for some minutes, care being necessary to avoid drying and crystallization of the stain on the slide. A period of 2 minutes is normally sufficient with fresh material, but longer times may be required with dry specimens. If overstaining occurs, the solution may be diluted with water, $1: 1$. The excess iodine is then shaken off and the slide pressure-dried between two pieces of filter paper (filter paper is preferable to blotting paper as the improved absorption minimizes crystallization). The smear is then covered with immersion oil, this acting as a clearing agent and delaying fading from sublimation of the iodine especially with fresh material. The slide may then be examined with either the $2 / 3^{\prime \prime}$ or the $1 / 12^{\prime \prime}$ oil-immersion objective, no cover-slip being required.

On completion of microscopy, the oil is removed by several washings with toluol and the slide may be kept for some months for repeat staining. Alternatively, 
decolourization before staining with Giemsa or Wright's stains is carried out by one of the following methods:

(i) Wash thoroughly in absolute or 95 per cent. methyl alcohol-which also acts as a pre-Giemsa fixative. The longer the slide is left stained with iodine the longer washing should be prolonged, up to several hours.

(ii) Wash in 0.5 per cent. solution of sodium hyposulphite for 10-15 minutes followed by prolonged washing in tap and distilled water. The first method is preferable, in that the addition of further chemicals which may crystallize is avoided, while at the same time the slide is fixed for further staining. Slides so decolourized may be re-stained with iodine several times, but after a period of weeks or months the staining becomes less certain, possibly owing to dessication. If decolourization is effectively carried out and not unduly delayed, no harmful effects on the subsequent use of other stains have been observed. Prolonged delay, particularly in fixation with alcohol, may result in unsatisfactory colour toning with the polychromatic stains.

\section{Examination}

The scraping as a whole is stained a fairly uniform golden-yellow against which, especially by condenser adjustments, the various types of cell-epithelial, mononuclear, polymorphonuclear cells, and red blood corpusclesmay be distinguished through the $1 / 12^{\prime \prime}$ objective by their size and nuclear outline.

HP inclusions, when present, stain a deep orange-brown and stand out clearly against the background, so that with a little experience they may readily be detected with the $2 / 3^{\prime \prime}$ objective, recourse being had to a higher power for confirmation. In detail they are seen as dotlike masses (although it is the matrix rather than the virus particles which is believed to stain with iodine) within the cytoplasm of epithelial cells, paranuclear but also often forming a network which encloses the nucleus. Rarely, an inclusion from a ruptured cell may be observed as an area of scattered staining dots in the vicinity of the affected cell.

In single and isolated cells, the structure of the inclusions may be studied in detail, and even when situated in a layer several cells thick the distinctive colouring and granularity are readily detected, although the finer detail may be obscure.

Intracellular melanin is visible as dark green-brown dots, coarser than the HP inclusions and usually of circum-nuclear distribution. Brown-staining clumps and crescents, non-granular in character and of a browner tint than the HP inclusions, may be present in the cytoplasm of polymorphonuclear cells. Crenated red blood corpuscles present a similar appearance. Occasionally, in the presence of severe bacterial and fungal infection, large clumps of amorphous staining material may be seen in the cytoplasm of large epithelioid cells which may be tissue macrophages; these clumps probably represent phagocytosis of degenerate leucocytic material. All these appearances may superficially resemble HP inclusions under the $2 / 3$ " objective, 
but the use of the $1 / 12^{\prime \prime}$ oil-immersion lens will disclose their true nature. Such identification can be made after considerably less experience than is required to identify HP inclusions in a Giemsa-stained preparation. Where serious doubt as to the nature of a staining object arises, the field should be located by vernier or "microviseur" readings and examined after restaining with Giemsa. However, not infrequently, the object cannot then be clearly distinguished against the deeply-stained background of a thick layer of tissue.

\section{Discussion}

Some of the particular advantages of the method have been discussed by Thygeson (1938) and it is felt that the modifications described accentuate these. They may be set out as follows:

(1) Speed, ease, and simplicity in staining and examination. With a little experience scrapings may be taken and stained, and an opinion on the presence or absence of inclusions given in 15 minutes. In consequence, the method would appear to have special advantages for field work.

(2) The ability to identify inclusions in thick smears, which would be missed by other methods. In our series of several hundred scrapings examined, between 25 and 30 per cent. of all inclusions seen would not have been visible in Giemsa preparations, and from 5 to 10 per cent. of all slides would have been labelled inclusion-negative.

(3) The morphology of the inclusions may be seen in greater detail, especially in regard to the relationship with other intracellular structures, e.g. nucleus, Golgi apparatus. Studies on this aspect of inclusion morphology might elucidate the peculiarly specific "helmet" shape which HP inclusions in general tend to assume.

The main disadvantage of the technique lies in the possibility that not all HP inclusions possess a glycogen-containing matrix, on the presence of which staining depends. It has been suggested that this may be absent in the extracellular phase and in the earliest stages of intracellular formation.

In practice it is doubtful whether this objection is valid. Smaller inclusions than may reliably be identified with Giemsa are readily seen, while the identification of extracellular virus particles by either method, except when in the vicinity of a ruptured inclusion containing cells, is a matter for uncertainty.

The question remains whether the method permits the visualization of initial body inclusions. Here the position is uncertain and will depend on the view taken of what is an initial body inclusion when Giemsa's stain is used.

Whether inclusions produced experimentally in the non-human conjunctiva, tissue culture, or yolk sac can be demonstrated with this technique is conjectural, but this would seem an appropriate subject for further study. 


\section{Conclusions}

The method described presents a simple way of staining and identifying the inclusion bodies of trachoma and inclusion conjunctivitis which should prove of especial value in field studies, particularly in connection with attempts at the culture of these viruses, so long as the only generally accepted evidence of infectivity is the presence of the specific HP inclusions. Similar considerations apply in connection with the conduct of therapeutic trials.

\section{Summary}

A modification of Rice's method (Rice, 1936) of staining the inclusion bodies of trachoma and inclusion conjunctivitis is described.

The potential advantages of this method over the Giemsa type stains recommended by the WHO Expert Committee on Trachoma, particularly for certain aspects of trachoma research, are discussed.

The facilities and assistance given by the Director and Staff of the Medical Research Council Laboratories, Fajara, Gambia, are gratefully acknowledged. Similar facilities were afforded by the Order of St. John of Jerusalem, in Jordan. Our thanks are due to Sir Stewart Duke-Elder, Honorary Director of the Group, for helpful advice and criticism.

\section{REFERENCES}

DARK, A. J. (1955). Brit. J. Ophthal., 39, 751.

GATENBY, J. B., ed. (1956). A. B. Lee, "The Microtomist's Vade Mecum", p. 43, para. 87. Churchill, London.

Julianelle, L. A. (1938). "The Aetiology of Trachoma”, p. 103-104. Commonwealth Fund, New York.

Rice, C. E. (1936). Amer. J. Ophthal., 19, 1.

Thygeson, P. (1938). Amer. J. Path., 14, 455

- (1955). WHO/Trachoma/55, p. 5. World Heath Organization, Geneva.

WORLD Health ORganization (1952). "Expert Committee on Trachoma. First Report". WHO/Trachoma/32, Annex II, p. 26.

(1955). "Expert Committee on Trachoma. Second Report". WHO/Trachoma/67, p. 14 , para. 6.1 .2 . 